\begin{tabular}{l|l|l|l} 
th $^{\text {th }}$ CONGIC & Blucher Design Proceedings \\
$7^{\text {th }}$ Information Design & $\begin{array}{l}\text { Setembro, 2015 - num. 2, vol.2 } \\
\text { proceedings.blucher.com.br }\end{array}$
\end{tabular}

\title{
A hierarquia da informação como facilitadora da navegação em plataformas de aprendizado online - Estudo de caso: MyEnglish Online
}

\author{
The information hierarchy as a tool for facilitating user navigation in online learning \\ platforms - Case study: My English Online
}

\author{
Vanessa M. Paulino, Walter G. Lima, Michelle B. Pedrosa, Erika S. Ferreira, Solange G. \\ Coutinho
}

\begin{abstract}
design da informação, hierarquia da informação, navegação, ensino a distância
Esta pesquisa apresenta um estudo de como uma estrutura informacional pode influenciar o desempenho de navegação de estudantes que utilizam plataformas de estudos online. Foi utilizado a plataforma de estudos My English Online - devido à significativa disponibilidade de usuários no contexto nacional e à atual relevância do estudo de idiomas. Por meio de uma pesquisa online, foram apontados, pelos usuários, alguns problemas observados na plataforma; dentre eles, destacamos a hierarquia da informação. $O$ método de Design Participativo (DP) - que consiste no envolvimento direto do usuário durante o processo de apreensão de dados -, foi usado como embasamento teórico. Foram criados dois mock-ups: o primeiro (mock-up-01) constituindo uma simulação da plataforma original, e o segundo (mock-up-02) trazendo uma nova proposta de organização informacional da plataforma. A partir da análise comparativa dos resultados, observou-se o impacto da hierarquia da informação exercida sobre a experiência do usuário - podendo servir como base para estudos futuros e melhoramentos de outras plataformas..
\end{abstract}

hierarchy, information, performance, online, studies

This research presents a study about how an informational structure can influence the performance of navigation of the students that use online platforms of studies. It was used the English language studies platform My English Online, due the greater viability of users and the studies of languages through online platforms become more popular and accessible. By meanings of an online research, it was point trouble for the participants of the platform. After analysis of researchers, the information's hierarchy was the focus of research. The method of Participative Design (PD) apprehension, which consists in the right involvement of the user during the process of apprehension of data, had been use for theoretical basis. There was create two mock-ups: the first (Mock-up-01) simulating the original platform and the second (Mock-up-02) bringing a new organization's proposal of the platform.

\section{Introdução}

Partindo do princípio de que só é informação aquilo que o usuário qualifica como tal (FREITAS et al, 2013), métodos de Design da Informação (Infodesign) são constantemente testados e analisados a fim de solucionar os problemas de comunicação. Como Design da Informação, entende-se a área do design que relaciona aspectos sintáticos, semânticos e pragmáticos organizando-os de forma concisa de acordo com seu público-alvo, e que tem como objetivo facilitar a comunicação (SBDI, 2014).

V. M. Paulino; W. G. Lima; M. B. Pedrosa; E. S. Ferreira; S. G. Coutinho. 2015. A hierarquia da informação como facilitadora da navegação em plataformas de aprendizado online - Estudo de caso: MyEnglish Online. In: C. G. Spinillo; L. M. Fadel; V. T. Souto; T. B. P. Silva \& R. J. Camara (Eds). Anais [Pôster] do $7^{\circ}$ Congresso Internacional de Design da Informação/Proceedings [Poster] of the 7th Information Design International Conference | CIDI 2015 [Blucher Design Proceedings, num.2, vol.2]. São Paulo: Blucher, 2015. ISSN 2318-6968, DOI 10.5151/designpro-CIDI2015-congic_35 
Alinhado a este conceito, Frascara (2000) aponta que "o objetivo de todo design de comunicação é a mudança que ocorre nas pessoas depois que a comunicação é apresentada". Existe também o proposto por Mijksenaar (1997, apud FRASCARA, 2000) que afirma que formas gráficas de representação podem ser apresentadas para provocar dinamismo e interesse nos usuários (QUINTÃO \& TRISKA, 2013).

Segundo dados informados pela Associação Brasileira de Ensino a Distância (ABED), em 2001, apenas 5.359 estudantes estavam matriculados na modalidade de ensino a distância (HIPÓLITO, 2012). Uma década depois, esse número aumentou 170 vezes - chegando a 930.179 alunos -, e já corresponde a 14,6\% do total das matrículas em cursos de graduação.

Diante desta realidade, foi definido como escopo de pesquisa as plataformas de ensino online; como recorte, as plataformas de língua estrangeira - devido à grande relevância destas, nos últimos anos.

Em um primeiro momento, uma pesquisa online no formato de questionário foi lançada para os estudantes da Universidade Federal de Pernambuco (UFPE) para mapear as plataformas mais utilizadas. Como resultado, teve $87,5 \%$ dos respondentes como usuários do site My English Online (MEO). Além desta referência, destaca-se as queixas dos usuários sobre inconveniências de diversas naturezas, presentes na referida plataforma - evidenciando a relevância de se antever erros de sistema e observar aqueles que podem ser resolvidos por meio do design da informação.

\section{A plataforma My English Online (MEO)}

O curso My English Online faz uso da plataforma MyELT e é utilizado, principalmente, por estudantes que desejam ingressar no programa Ciência Sem Fronteiras. Além de livros interativos, dicionários, textos e vídeos da Revista National Geographic, o aluno pode usufruir de atividades teóricas ou orais.

\section{O problema}

Dentre os problemas apresentados pelos usuários que responderam ao questionário online, a hierarquia da informação - na disposição dos links e agrupamentos de atividades no site - foi um fator preponderante. Observamos que os exercícios são organizados de acordo com o tipo de atividade que o indivíduo deseja realizar. (Figura 1).

Figura 1: Página de navegação de exercícios do MyELT

Anais [Pôster] do $7^{\circ}$ Congresso Internacional de Design da Informação | CIDI 2015

Proceedings [Poster] of the 7th Information Design International Conference | IDIC 2015 


\section{MyELT}

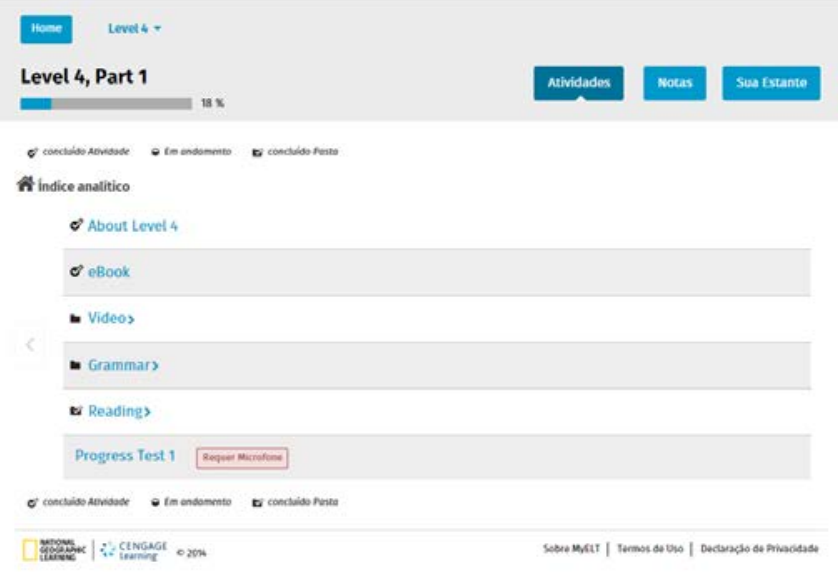

Na interface que se sucede à escolha do tipo de atividade, são apresentadas ao usuário as unidades disponíveis (figura 2) para que, após sua seleção, seja possível o acesso às diferentes lições do curso (figura 3).

Figura 2: Página dos exercícios de vídeos da página do MyELT.

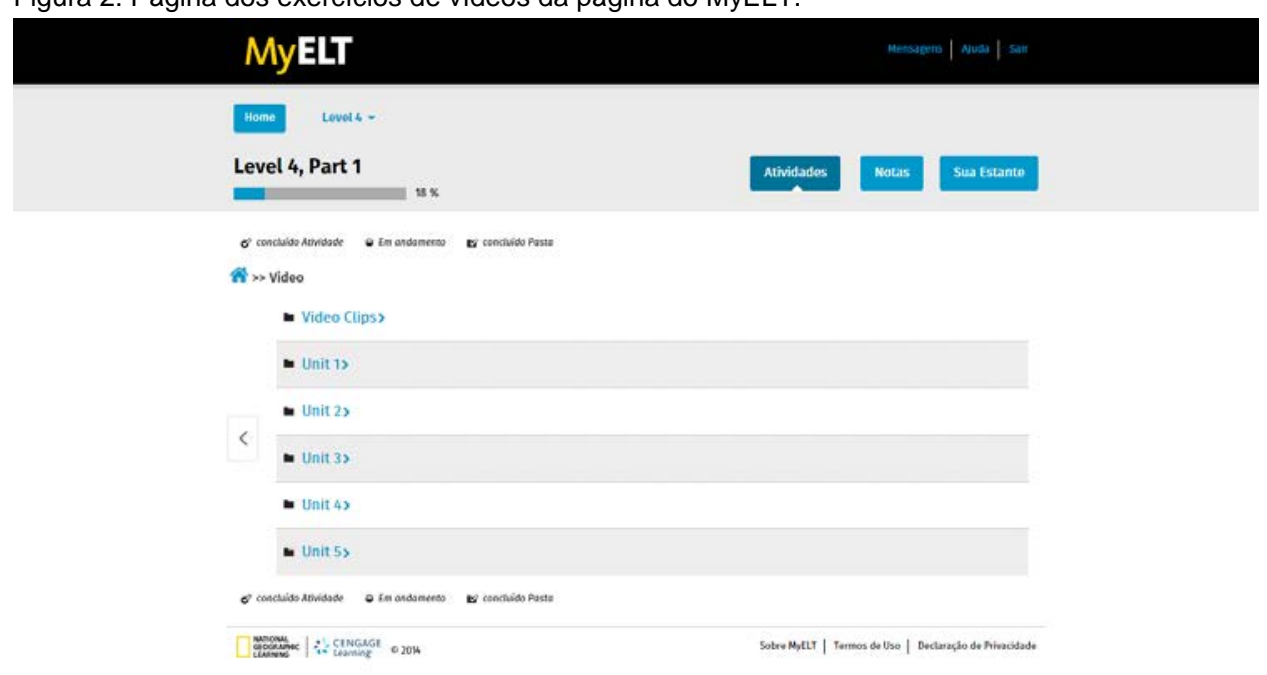

Figura 3: Página da unidade 1 dos exercícios de vídeo do MyELT. 


\section{MyELT}

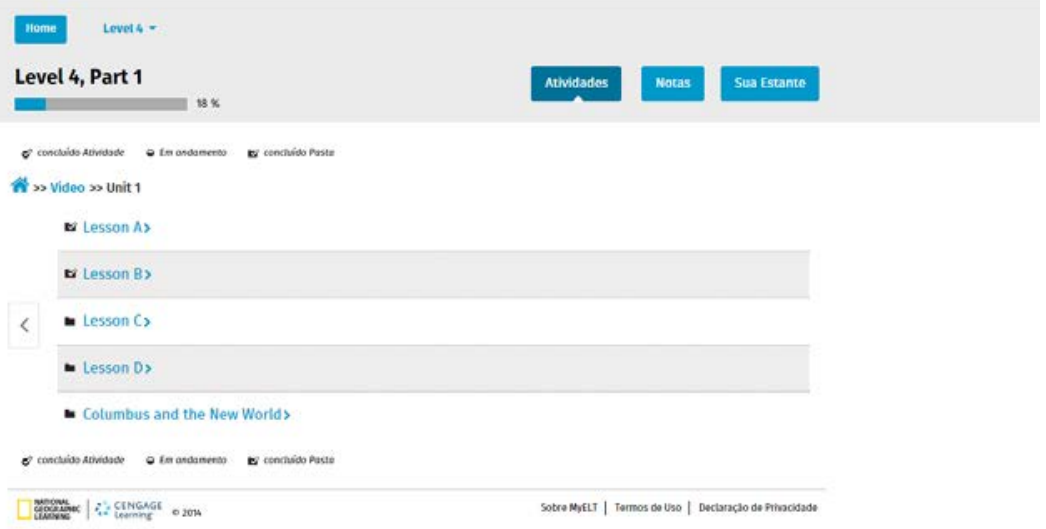

Ao analisar esta estrutura, pode-se observar uma necessidade de o estudante precisar voltar à página principal sempre que desejar acessar uma atividade diferente de uma mesma unidade. Por exemplo: caso o estudante queira completar todas as atividades referentes à unidade 1 antes de ir para a unidade 2, ele terá que, ao fim de cada tipo de atividade (vídeo, reading, etc), retornar à página inicial para escolher um tipo de atividade diferente, referente à unidade 1. Isso poderia acarretar em:

- Transtorno na velocidade e facilidade de navegação;

- Quebra do ritmo de estudo do usuário devido à necessidade de situar-se novamente à interface, após o término de uma atividade;

- Indução à realização de atividades de outras unidades, antes de terminar a unidade em questão - de acordo com a programação da plataforma; e

- Confusão na navegação - devido ao grande número de atividades de diferentes unidades próximas umas das outras;

Assim, através de um experimento realizado com o público-alvo da plataforma, foi proposta uma nova hierarquia informação - reorganizando as atividades das plataformas e suas respectivas unidades. Em um primeiro momento, os usuários acessariam as unidades (Units); depois, poderiam escolher quais atividades gostariam de praticar (figuras 4,5 e 6).

Figura 4: Navegação dos exercícios do MyELt Modificada. 


\section{MyELT}

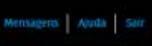

tomentis

Level 4, Part 1

$18 \mathrm{x}$

Mividades Motas Sua tstante

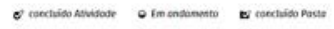

ศindice analitico

about Level 4

o eBook

- Reading

- Unit 1

- Unit 2

- Unit 3

- Unit 4

- Unit 5

Progress Test 1 cons rons

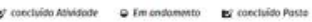

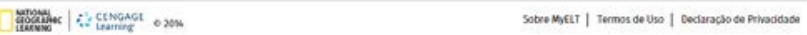

Figura 5: Página Unit do MyELT modificada.

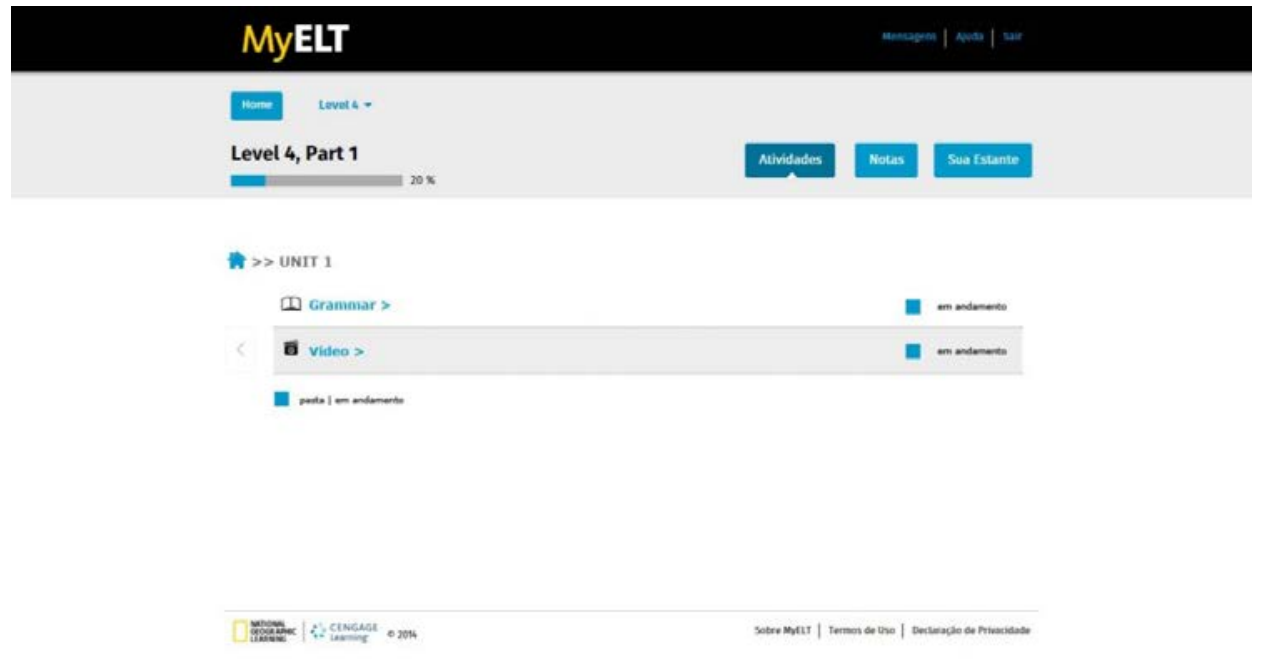

Figura 6: Página Grammar do MyELT modifica

Anais [Pôster] do $7^{\circ}$ Congresso Internacional de Design da Informação | CIDI 2015

Proceedings [Poster] of the 7th Information Design International Conference | IDIC 2015 


\section{MyELT}

towet

Level 4, Part 1

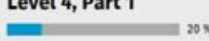

Mividades Notas Sua bsumto

h $>>$ UNIT $1 \gg>$ Grammar

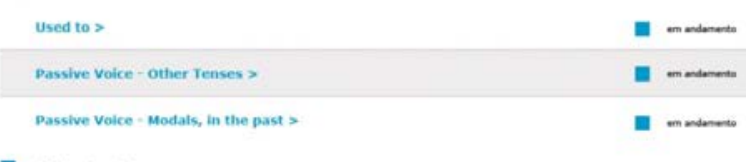

घ

andences

\section{Metodologia e resultados}

Com o objetivo de investigar as dificuldades de navegação da plataforma do My English Online, foi utilizado o método de Design Participativo (DP), que consiste no envolvimento direto do usuário durante o processo de apreensão de dados. Nesse caso, utilizamos mock-ups para obter informações.

Para os experimentos, participaram estudantes da Universidade Federal de Pernambuco (UFPE), não familiarizados com o layout do MEO e com diferentes níveis de inglês. Os mock-ups foram desenvolvidos a partir do layout das atividades do nível 4 da plataforma - nível com maior conteúdo didático disponível.

Figura 7: Página Grammar do MyELT modificada

\section{MyELT}

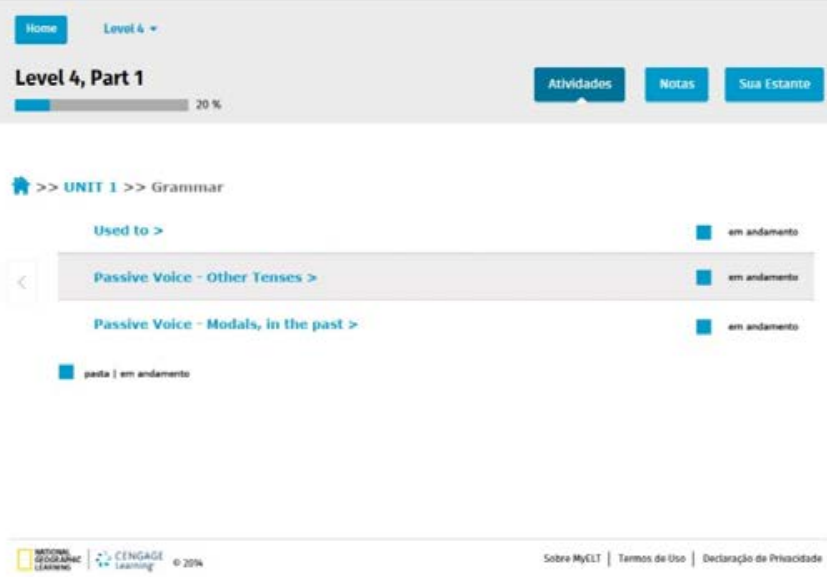

Anais [Pôster] do $7^{\circ}$ Congresso Internacional de Design da Informação | CIDI 2015

Proceedings [Poster] of the 7th Information Design International Conference | IDIC 2015 
Figura 8: Questionário para Mock-Ups 1A e 2A

\section{Questionário, Mock-Up A}

Qual o seu nivel de conhecimentos em Lingua Inglesa?
[ ] Básico [ ] Pré-intermediário [ ] Intermediário
[ ] Avançado
Qual a sua idade?
[ ] 14 a 16 anos [ ] 17 a 21 anos
[ ] 22 a 30 anos [ ] 31 anos ou mais

Esta é uma simulação da página MyElt, relacionada ao curso My English Online, pedimos para que você cumpra as missões na página do MyELT.

MISSŐES:

1. Localize o Lesson A da Unit 05 .
Você conseguiu executar esta tarefa? [ ] Sim [ ] Não

1.1 Localize o conteúdo Present Perfect Form of Modals da Unit 03.

Você conseguiu executar esta tarefa? [ ] Sim [ ] Não

1.2 Localizar o Video Clip da Unit 03.

Você conseguiu executar esta tarefa? [ ] Sim [ ] Não

1.3 Localize a atividade Pronunciation Practice em Unit

03, Lesson B; e em seguida informe que material é

solicitado pelo site para realização dessa atividade:

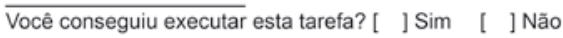

Figura 9: Questionário para Mock-Ups 1B e 2B.

\section{Questionário, Mock-Up B}

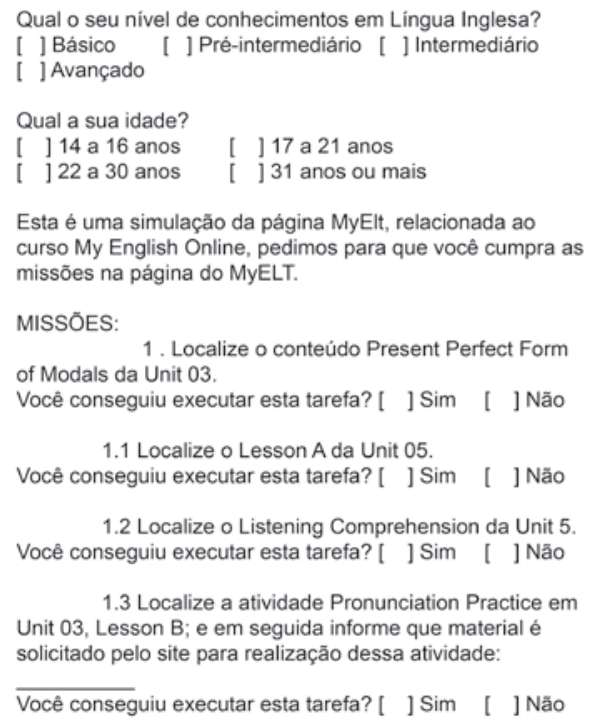

Unit 5.

$$
\text { 1.4 Localize o Listening Comprehension da }
$$

Você conseguiu executar esta tarefa? [ ] Sim [ ] Não

\author{
1.5 Localize o texto para leitura One Boy's \\ Journey \\ Você conseguiu executar esta tarefa? [ ] Sim [ ] Não
}

Questionário após a realização das tarefas na plataforma do My English Online:

1. Sente-se confortável/familiarizado com a ordem das etapas apresentadas no site?

2. Em que nível de dificuldade você enquadraria a realização dos exercicios solicitados? [ ] Muito Fácil [ ]Fácil [ ] Médio [ ] Dificil [ ] Muito dificil

3.Por que você atribuiu esse nível de dificuldade?

4) Por favor, faça um comentário sobre o site. Críticas e sugestões são bem-vindas!

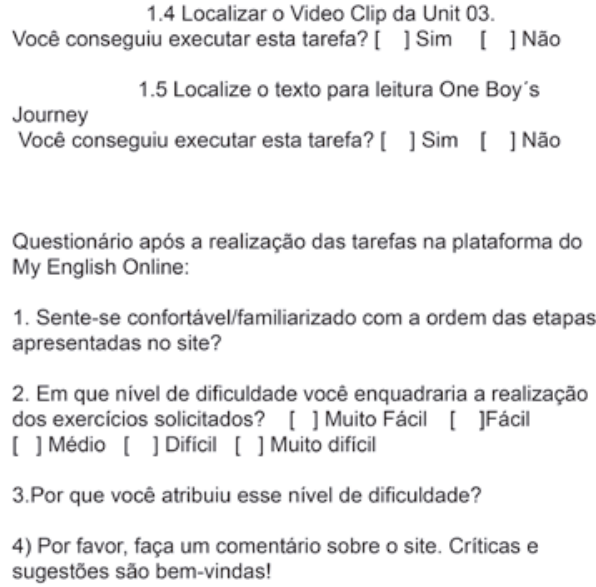

1. Sente-se confortável/familiarizado com a ordem das etapas apresentadas no site?

2. Em que nivel de dificuldade você enquadraria a realização dos exercícios solicitados? [ ] Muito Fácil [ ]Fácil [ ] Médio [ ] Difícil [ ] Muito difícil

3.Por que você atribuiu esse nivel de dificuldade?

4) Por favor, faça um comentário sobre o site. Criticas e sugestões são bem-vindas!

\section{Piloto}

Para início da pesquisa, a equipe criou o mock-up-01 - réplica do site MEO - e um guia de tarefas com diretrizes para experimentação do site, com perguntas acerca de seu uso. Nesta etapa, participaram 15 voluntários, ela tinha como objetivo testar o mock-up-01 e a metodologia de pesquisa, identificando possíveis falhas.

Como alteração, para o experimento, passou-se a solicitar o nível de inglês dos usuários e dividir o guia de tarefas em duas versões: A e B, com a ordem das perguntas diferentes, a fim 
de verificar se esta alteração influenciaria na realização das tarefas. Para obter as imagens das telas dos computadores e a voz dos participantes, foi utilizado o programa "Camtasia Studio".

\section{Experimento}

\section{Grupo Controle}

Nesta etapa, foi aplicado o mock-up-01 em duas versões, 1A e 1B, em um grupo de 10 pessoas. Ao término das tarefas, foi realizada uma entrevista semiestruturada com cada usuário, a fim de saber o nível de dificuldade atribuído à resolução de cada exercício.

Após análise e descrição das ações dos usuários, computamos o número de cliques que cada pessoa utilizou até concluir as atividades (figura 10).

Figura 10: Grupo controle.

\section{Grupo Controle \\ COMPARATIVO}

Mock-Up IA Mock-Up IB

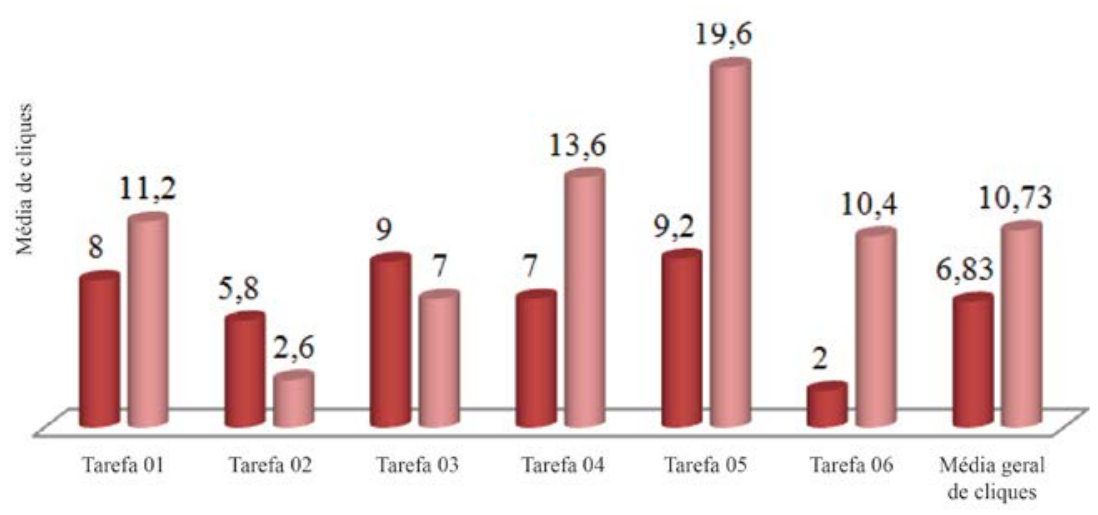

Desta forma, o gráfico da figura 10 apresenta a média de cliques por tarefa solicitada, a diferença dos resultados quando modificada a ordem das tarefas em cada guia (A e B) e, por último, a média aritmética de cliques das tarefas 1A e 1B.

Pode-se observar que os usuários que responderam ao questionário 1B fizeram uso de, aproximadamente, o dobro de cliques do que os usuários que utilizaram o $1 \mathrm{~A}$.

Grupo Experimental

Após análise dos resultados do Grupo Controle, foram identificados os principais problemas da plataforma em relação à experiência dos usuários observados. Dessa forma, foi construído o mock-up-02, com as propostas de melhorias no design da informação. Seguindo os mesmos parâmetros do experimento com o Grupo Controle, foi testada a usabilidade do novo mock-up, com participação de 10 voluntários.

Destacamos que, basicamente, a diferença entre os mock-ups 1 e 2 é a de que os conteúdos foram estruturados por meio de unidades - no lugar de áreas, como Grammar e Speaking - e foram colocados ícones para que os usuários pudessem perceber com mais facilidade as áreas de conhecimento. 
Figura 11: Grupo Experimental.

\section{Grupo Experimental \\ COMPARATIVO}

Mock-Up 2A Mock-Up 2B

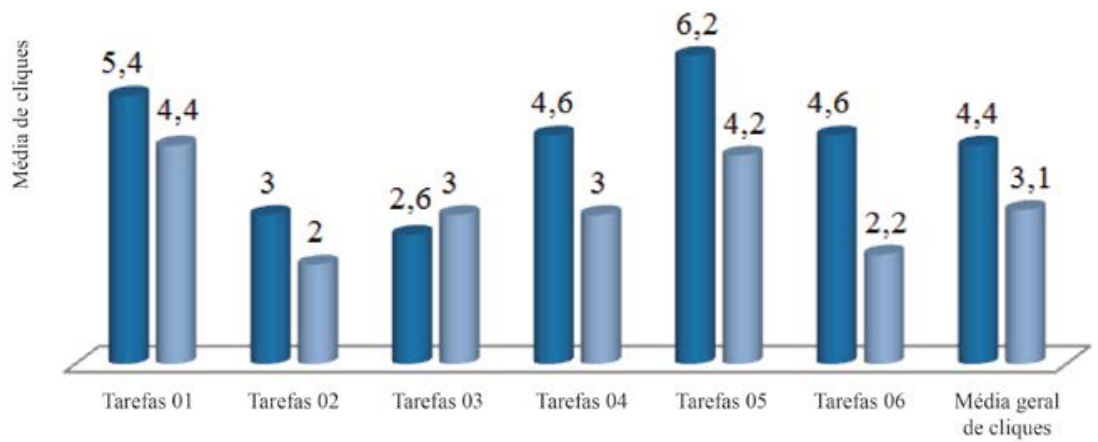

Observa-se que a diferença na média de cliques dos usuários $2 \mathrm{~A}$ e $2 \mathrm{~B}$ é mínima comparada aos Mock-Ups 1A (6,83 cliques) e 1B (11,58 cliques). Sendo assim, supõe-se que a ordem das perguntas não alterou de forma significativa os resultados, evidenciando uma maior facilidade de navegação do usuário.

Os participantes também levaram menos tempo para executar o que lhes era pedido. A maioria atribuiu como fácil o nível de dificuldade para execução das atividades e mencionaram que os ícones ajudaram no trajeto até encontrarem o conteúdo.

\section{Conclusão}

Ao comparar os resultados dos usos dos diferentes mock-ups, por meio da contabilização dos cliques, observa-se que o re-design apresentou resultados muito positivos (figura 12).

Figura 12: Comparativo.

\section{Grupo Controle vs. Grupo Experimental}

Grupo controle $\square$ Grupo experimental

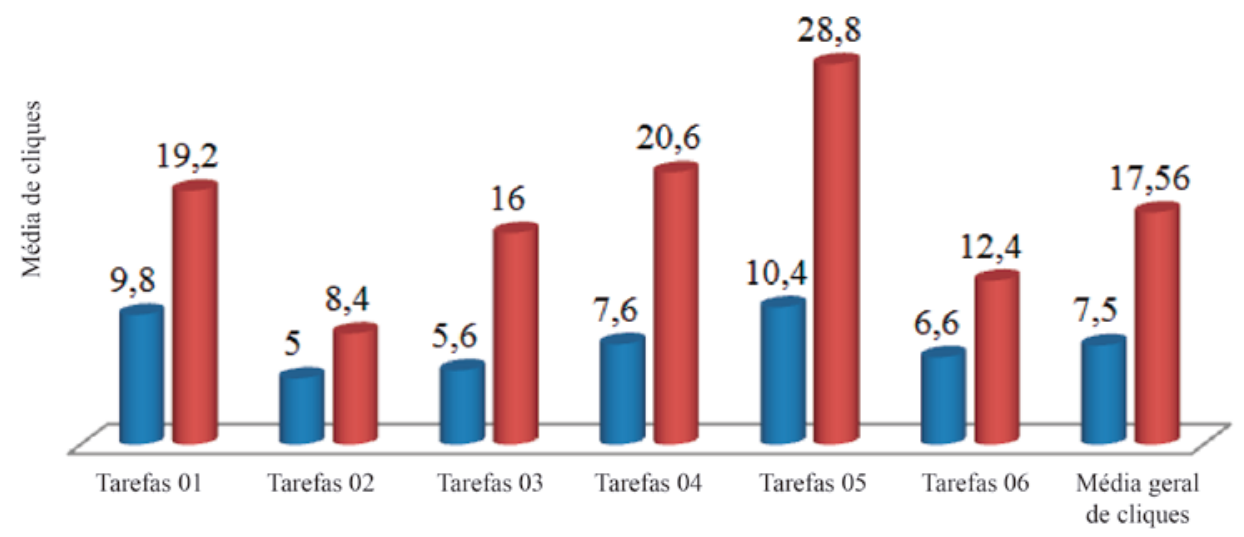

Anais [Pôster] do $7^{\circ}$ Congresso Internacional de Design da Informação | CIDI 2015

Proceedings [Poster] of the 7th Information Design International Conference | IDIC 2015 


\section{Agradecimentos}

Agradecemos a Daniel Quintino e Rebeca Ribeiro pela colaboração no desenvolvimento da pesquisa, no que diz respeito a ajuda na coleta dos dados.

\section{Referências}

FRASCARA. J. 2002. Diseño gráfico para la gente: comunicaciones de massa y cambio social. Buenos Aires: Ediciones Infinito.

HIPÓLITO, O. 2012. Educação a distância: uma nova realidade. Estadão, São Paulo, 31 mai. 2012. Disponível em: http://educacao.estadao.com.br/noticias/geral,educacao-a-distanciauma-nova-realidade,880620 - acesso 13/1/ 2015.

MIJKSENAAR, P. 1997. Visual function: an introduction to information design. Rotterdam: 010 Publishers.

QUINTÃO, F. S.; TRISKA, R. 2013. Design de informação em interfaces digitais: origens, definições e fundamentos. Revista Brasileira de Design da Informação. São Paulo: v. 10, n. 2.

SANTA ROSA, J. G.; GURGEL, A.; PASSOS, M. 2012. Técnicas baseadas em etnografia e prototipagem no design de interface de aplicativo mobile para gerenciamento acadêmico. InfoDesign, São Paulo, v. 9, n. 2.

SBDI: SOCIEDADE BRASILEIRA DE DESIGN DA INFORMAÇÃO. 2014. In: Home: SBDI. Disponível em: http://www.sbdi.org.br - acesso 01/1/ 2015.

ZAVANELLA, V. 2013. MEO - MEO - My English Online - curso de inglês online gratuito oferecido pelo MEC e Capes.

\section{Sobre os autores}

Michelle Bittencourt Pedrosa, Graduando em Design, UFPE, Brasil <michbittencourt@hotmail.com >

Vanessa Martins Paulino, Graduando em Design, UFPE, Brasil: <vanmartinsp@gmail.com>

Walter Gabriel Melo de Lima, Graduando em Design, UFPE, Brasil

$<$ waltergabriel@hotmail.com.br>

Erika Simona dos Santos Ferreira, Mestranda em Design, UFPE, Brasil

<erikasimona@gmail.com>

Solange Galvão Coutinho, Doutora,UFPE, Brasil <solangecoutinho@globo.com> 